\title{
Therapy with un-engineered naïve rat umbilical cord matrix stem cells markedly inhibits growth of murine lung adenocarcinoma
}

\author{
Dharmendra K Maurya, Chiyo Doi, Atsushi Kawabata, Marla M Pyle, Clay King, Zhihong Wu, Deryl Troyer, \\ Masaaki Tamura*
}

\begin{abstract}
Background: Lung cancer remains the leading cause of cancer-related mortality despite continuous efforts to find effective treatments. Data from the American Cancer Society indicate that while the overall incidence of lung cancer is declining, it continues to rise in women. Stem cell-based therapy has been an emerging strategy to treat various diseases. The purpose of this paper is to determine the efficacy of an intrinsic anti-cancer effect of rat umbilical cord matrix stem cells (UCMSCs) on lung cancer.
\end{abstract}

Methods: A mouse syngeneic lung carcinoma model was used to test the basic ability of UCMSCs to control the growth of lung cancer. Lung tumors were experimentally induced by tail vein administration of Lewis lung carcinoma (LLC) cells derived from the lung of C57BL/6 mouse. Rat UCMSCs were then administered intratracheally five days later or intravenously on days 5 and 7 . The tumor burdens were determined by measuring lung weight three weeks after the treatment.

Results: Co-culture of rat UCMSCs with LLC significantly attenuated the proliferation of LLC cells as monitored by MTT (3-(4,5-Dimethylthiazol-2-yl)-2,5-diphenyltetrazolium bromide), a tetrazole cell proliferation assay, thymidine uptake, and direct cell counts. In vitro colony assays with rat UCMSCs as feeder layers markedly reduced LLC colony size and number. Co-culture of rat UCMSCs with LLCs causes G0/G1 arrest of cancer cells. This is evident in the decrease of cyclin A and CDK2 expression. The in vivo studies showed that rat UCMSC treatment significantly decreased tumor weight and the total tumor mass. Histological study revealed that intratracheally or systemically administered rat UCMSCs homed to tumor areas and survived for at least 3 weeks without any evidence of differentiation or adverse effects.

Conclusions: These results indicate that rat UCMSCS alone remarkably attenuate the growth of lung carcinoma cells in vitro and in a mouse syngeneic lung carcinoma graft model and could be used for targeted cytotherapy for lung cancer.

\section{Background}

Despite rapid advances in diagnostic and operative techniques, lung cancer remains one of the most difficult human malignancies to treat. The American Cancer Society estimates that 214,440 persons in the United States developed lung cancer in 2009, with 159,390 deaths [1]. Lung cancerdependent deaths constituted 30\% (men) and 26\% (women) of the estimated total cancer-related deaths in

\footnotetext{
* Correspondence: mtamura@vet.ksu.edu

Department of Anatomy \& Physiology, Kansas State University, College of Veterinary Medicine, Manhattan, KS 66506, USA
}

2009 [1]. Data from the American Cancer Society indicate that while the overall incidence of lung cancer is declining, it continues to rise in women [1]. The possible treatments for lung cancer include surgical resection, chemotherapy, radiotherapy, and/or combination therapy. Recently, multiple new chemotherapeutic agents have been developed and some are in clinical trials [2,3]. Although some of these have produced promising results, their therapeutic spectrum is narrow.

Genetically engineered mesenchymal stem cells derived from the umbilical cord matrix have great 
potential for therapeutic application in various diseases including cancer. However, a major advantage would be realized if tumor-trafficking stem cells that have not been genetically modified exhibit an inherent anti-tumor effect, thus circumventing the necessity of the expression of exogenous genes by the cells. It is well known that stem cells have inherent tumoritropic migratory properties [4]. Signals that mediate this effect appear to be similar or identical to those that mediate recruitment of stromal or defensive cells in tumors [5-7]. There are also a number of reports showing that genetically engineered stem cells efficiently deliver therapeutic proteins to cancer and other sites of inflammation [4,6,8-12]. Stem cells we have isolated from the Wharton's jelly of umbilical cord, termed 'umbilical cord matrix stem cells' (UCMSCs), also exhibit inherent tumoritropic migratory properties [8].

UCMSCs may be more useful for cancer therapy than other adult stem cells, since they are easy to prepare in relatively large quantities from umbilical cords after delivery and pose no ethical issues. They are potentially quite applicable to human patients without a complete genetic match, since they are unlikely to induce an acute immune response $[13,14]$. The versatility and availability of umbilical cord stem cells makes them a potent resource for transplant therapies for various diseases, including cancer. When these cells are engineered to secrete a cytokine, interferon beta (IFN- $\beta$ ), and are administered intravenously, they can attenuate metastatic breast cancer in a SCID mouse model [8]. Recently we found that rat UCMSCs completely abolished the growth of Mat B III cancer cells in vitro and in vivo [15]. Furthermore, un-engineered human UCMSCs have been shown to attenuate human breast cancer xenografts in a SCID mouse model [16]. Accordingly, the primary objective of the present study was to explore the therapeutic potential of rat UCMSCs against lung cancer using an LLC tumor model in syngeneic immunocompetent mice. This study surprisingly indicated that, even in trans-species transplantation, rat UCMSCs have exhibited a profound anti-tumor effect on lung carcinoma without any significant rejection of transplanted rat UCMSCs.

\section{Methods}

\section{Cell culture}

Rat UCMSCs were prepared from E19 pregnant rats and isolated using the method described previously [15]. Cells were maintained in defined medium, containing a mixture of 56\% low glucose Dulbecco's Modified Eagle Medium (DMEM, Invitrogen, CA); 37\% MCDB 201 (Sigma; St. Louis, MO); $2 \%$ fetal bovine serum (FBS, Atlanta Biologicals Inc, GA); 1x insulin-transferrin-selenium-X (ITS-X, Invitrogen); 1x ALBUMax1 (Invitrogen); $1 \mathrm{x}$ penicillin/streptomycin (Pen/Strep, Invitrogen);
$10 \mathrm{nM}$ dexamethasone (Sigma); $100 \mu \mathrm{M}$ ascorbic acid 2-phosphate (Sigma); $10 \mathrm{ng} / \mathrm{ml}$ epidermal growth factor (EGF, R\&D systems, Minneapolis, MN); and $10 \mathrm{ng} / \mathrm{ml}$ platelet derived growth factor-BB (PDGF-BB, R\&D systems). Cells were maintained at $37^{\circ} \mathrm{C}$ in a humidified atmosphere containing $5 \% \mathrm{CO}_{2}$.

The LLC cell line was maintained in DMEM (Invitrogen) medium supplemented with $10 \%$ FBS and $1 x$ Pen/ Strep (Invitrogen). Cells were cultured at $37^{\circ} \mathrm{C}$ in a humidified atmosphere containing $5 \% \mathrm{CO}_{2}$.

\section{Cell proliferation assay}

The MTT assay was performed to study the effect of rat UCMSCs on LLC cell proliferation. In brief, different ratios of rat UCMSCs and LLCs (UCMSCs:LLCs = 1:10, $1: 6$, and 1:3) in DMEM with 10\% FBS were seeded in 96 well plates; cells were allowed to grow for $72 \mathrm{hrs}$. MTT solution $(20 \mu \mathrm{l}$ of $5 \mathrm{mg} / \mathrm{ml})$ was added $4 \mathrm{hrs}$ before completing 72 hrs of incubation. Formazan crystals formed were dissolved by adding $100 \mu \mathrm{l}$ solublization buffer $(10 \%$ SDS containing $0.01 \mathrm{~N} \mathrm{HCl})$ and incubated overnight at $37^{\circ} \mathrm{C}$. The following day, color developed was measured at $550 \mathrm{~nm}$ and background absorbance was measured at $630 \mathrm{~nm}$ using the Molecular Devices Spectramax 190 plate reader (Global Medical Instrumentation, Inc. Ramsey, MN).

\section{$\left[{ }^{3} \mathrm{H}\right]$ thymidine uptake assay}

To evaluate cell proliferation from a different angle, a $\left[{ }^{3} \mathrm{H}\right]$ thymidine uptake assay was carried out. In brief, rat UCMSCs $\left(1 \times 10^{3}\right.$ or $2 \times 10^{3} /$ well $)$ were mixed with $6 \times 10^{3}$ LLC cells/well, directly plated in 24-well culture plates, and cultured in a $\mathrm{CO}_{2}$ incubator for $72 \mathrm{hrs}$. Radioactivity incorporated into the cells was analyzed using our published protocol[15].

\section{Transwell cell culture study}

Direct cell counts via hemocytometer were performed to study the effect of rat UCMSCs on LLC cell growth in culture plates with cell culture inserts (BD Biosciences, San Jose, CA). In brief, LLC cells were seeded in normal growth medium at $1 \times 10^{5}$ cells/well in 6 -well plates. After allowing the cancer cells to adhere for $1 \mathrm{hr}, 1.67 \times$ $10^{4}$ and $3.33 \times 10^{4}$ rat UCMSCs were seeded on the cell culture inserts $(3.0 \mu \mathrm{m}$ pore size). The insert pore size of $3 \mu \mathrm{m}$ is small enough to prevent cells migrating from the inserts to the culture dishes, since UCMSCs do not penetrate this size pores in the insert. After $72 \mathrm{hrs}$ co-culture, cells grown in the bottom culture dish were collected by trypsinization and counted using a hemocytometer.

\section{Colony formation study}

Various numbers of rat UCMSCs $\left(8.33 \times 10^{2}\right.$ and $1.67 \times$ $10^{3}$ cells/well) were grown in a 12 well tissue culture 
plate. One day after seeding, $0.75 \mathrm{ml} 0.8 \%$ agar (Sea Plaque agar, Cambrex Bio Science Rockland, Inc. Rockland, $\mathrm{ME}$ ) in DMEM with $10 \%$ FBS was poured into the dish (bottom layer). After the bottom agar layer solidified, LLC cells $\left(5 \times 10^{3}\right.$ cells) were suspended in $0.5 \mathrm{ml}$ of DMEM containing $10 \%$ FBS and $0.5 \%$ agar and plated on top of the bottom agar layer. The cells were incubated at $37^{\circ} \mathrm{C}$ with $5 \% \mathrm{CO}_{2}$ for growth of colonies. On day 16 , colony growth was evaluated by an automated phase contrast microscope equipped with Micro Suite Analysis Suite (Olympus CKX41, Center Valley, PA). Colonies with an area greater than $50000 \mu \mathrm{m}^{2}$ were counted using Micro Suite Analysis Suite software.

\section{Cell cycle analysis}

To analyze the effect of rat UCMSC co-culture on LLC cells, cell cycle analysis was carried out using propidium iodide staining. In brief, rat UCMSCs and LLC cells were co-cultured in 6 well Transwell culture dishes as described in the Transwell cell culture study. At the end of incubation LLC cells in the bottom chamber were collected and analyzed for cell cycle using our standard protocol [16].

\section{Western blot analysis}

Total cellular protein was prepared using lysis buffer (1\% TritonX-100, 0.1\% SDS, 0.25M sucrose, $1 \mathrm{mM}$ EDTA, $30 \mathrm{mM}$ Tris- $\mathrm{HCl}$ (pH 8.0)) supplemented with protease inhibitor cocktail (Boehringer Mannheim, Indianapolis, IN). Protein samples were separated by a 10\% SDS-PAGE gel, electroblotted onto nitrocellulose membrane (GE Healthcare, Uppsala, Sweden) and blocked with $4 \%$ nonfat dry milk in $0.1 \%$ Tween 20 in phosphate buffered saline (PBST) for $1 \mathrm{hr}$ at room temperature. The membranes were washed and incubated with antibodies against cyclin A (1:100, Abcam, Cambridge, MA), cyclin E (1:100, Abcam), and CDK2 (1:100, Santa Cruz Biotechnology, Santa Cruz, CA) with $4 \%$ nonfat dry milk in PBST for $1 \mathrm{hr}$ at room temperature and then with a horseradish peroxidase-conjugated anti-rabbit IgG secondary antibody (GE Healthcare). The protein expression signal was detected with Pierce ECL Western Blotting substrate (Pierce, Rockford, IL). GAPDH was used as the loading control of sample by reprobing with an anti-GAPDH antibody (1:4000, Santa Cruz Biotechnology).

\section{Animals}

Wild-type female C57BL/6 mice were obtained from the Jackson Laboratory (Bar Harbor, ME). All mice were housed in an AAALAC-accredited clean facility and held for 10 days to acclimatize. All animal procedures received prior approval from the Kansas State University Institutional Animal Care and Use
Committee (protocol \# 2681) and were performed in adherence with all applicable international, federal, state, and local guidelines.

\section{Systemic transplantation of LLC in lung and UCMSC treatment}

To study the effect of rat UCMSCs on the growth of lung cancer grafts, a syngeneic LLC tumor model was used. In brief, each mouse was injected via the tail vein with $1.5 \times 10^{6}$ LLC cells (for Experimental design I) or $2 \times 10^{6}$ cells (for Experimental design II) suspended in $200 \mu \mathrm{l}$ of PBS. On day 5 all mice were randomized into two treatment groups: a PBS control group and a rat UCMSC treatment group. To evaluate the trafficking of transplanted UCMSCs, cells were labeled with $10 \mu \mathrm{g} / \mathrm{ml}$ of SP-DiI fluorescent dye (Molecular Probes, CA) for 16 hrs incubation in a $5 \% \mathrm{CO}_{2}$ incubator. After removing excess dye by washing the cells with PBS, cells were incubated with dye-free medium for another $4 \mathrm{hrs}$. These cells were dispersed by trypsinization and mixed with unlabeled UCMSCs so that $20 \%$ of the UCMSCs were labeled with SP-DiI. Although a preliminary cell culture study indicated that SP-DiI does not alter viability of UCMSCs, only $20 \%$ of transplanted cells were labeled in order to minimize any potential adverse effects of the dye. Two experimental designs were used for UCMSC injections.

\section{Experimental design I}

five days after LLC cell injection, these mice were injected intratracheally using a 27 gauge needle with either $35 \mu \mathrm{l}$ PBS $(\mathrm{n}=8)$ or $35 \mu \mathrm{l}$ rat UCMSCs suspension $\left(3.5 \times 10^{5}\right.$ cells in PBS, $\left.\mathrm{n}=9\right)$. The cell suspension in the syringe was released at approximately $5 \mathrm{~mm}$ above the dividing point of the bronchi. Our preliminary evaluations have shown that this injection method evenly distributes injected cells into the right and left lobes of the lung. Experimental design II: On day 5 and day 7 after LLC injection, these mice were systemically injected using a 28 gauge needle with either $200 \mu \mathrm{l}$ PBS $(\mathrm{n}=8)$ or $200 \mu \mathrm{l}$ UCMSCs suspension $\left(1 \times 10^{6}\right.$ cells in PBS, $n=8$ ) through the tail vein (IV).

All mice were kept in their cages and their physical condition was monitored until sacrifice on day 21 after LLC transplantation. Lungs of mice were collected immediately after sacrifice without inflation treatment, fixed in $10 \%$ formalin in saline, and used for histochemical analysis.

\section{Histopathology}

Lung tissue fixed in $10 \%$ formalin in saline was embedded in paraffin, serially sectioned at $4 \mu \mathrm{m}$, and stained with hematoxyline and eosin (H\&E) for microscopic examination of morphology of tumors. Unstained 
serial paraffin sections were counter stained with DAPI for nuclei and observed under epifluorescence microscopy for tracking the rat UCMSCs.

\section{Detection of apoptosis in tumors}

To determine apoptosis in the tumors, the DeadEndTM colorimetric TUNEL system (Promega, Madison, WI) was used according to the manufacturer's protocol with a slight modification. The sections were counterstained with methyl green. To determine the apoptotic index, 10 nodules were selected randomly by light microscopy and the area of TUNEL positive cells in each nodule was calculated using the NIH Image J analysis software. The index was assessed as the percentage of TUNELpositive area in the tumor. The fold change was calculated by dividing TUNEL-positive area in rat UCMSC treated tumors by those in untreated tumors.

\section{Statistical analysis}

All values are expressed as means \pm SE. For all in vitro and in vivo experiments, statistical significance was assessed by Tukey-Kramer Pairwise Comparisons test. All experiments were conducted at least twice with multiple sample determinations. Actual number of experiments repeated and sample numbers/experiments are described in the figure legends. Since all experiments were very closely reproduced, raw values were used for the statistical analysis. Data from replicate experiments as well as replicates within experiments were combined with no for experiment. A value of $\mathrm{P}<0.05$ was considered significant.

\section{Results}

Rat UCMSCs inhibit anchorage-dependent and -independent growth of LLC cells

The effect of un-engineered rat UCMSCs on anchoragedependent growth of LLC cells was evaluated by both direct and indirect co-culture. As shown in Figure 1, direct co-culture of rat UCMSCs with LLC cells (ratio, 1:6 or 1:3) markedly decreased the total LLC cell number as measured by MTT assay. DNA synthesis as determined by thymidine uptake assay was in good agreement with the MTT assay result (Figure 2), although significant growth inhibition of LLC cells by rat UCMSCs was observed with a smaller proportion of UCMSCs in the MTT assay (UCMSC:LLC $=1: 6$ ) than in the thymidine uptake assay (UCMSC:LLC $=1: 3$ ). In addition to these studies in which both cell types directly contacted each other, an indirect co-culture study was carried out using a Transwell culture system in which rat UCMSCs were cultured in inserts and LLC cells were cultured in the bottom of the well. The effect of this indirect co-culture was assessed by counting the LLC cells after $72 \mathrm{hrs}$ coculture. The findings of this experiment (Figure 3) also

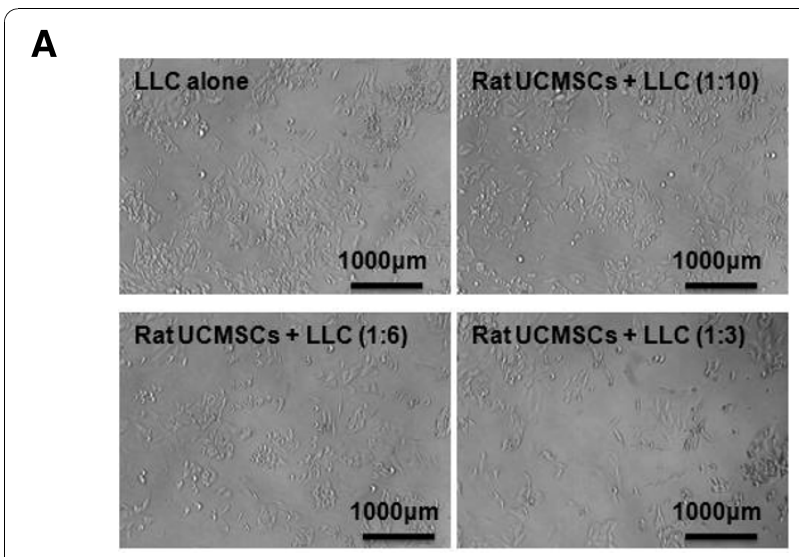

B

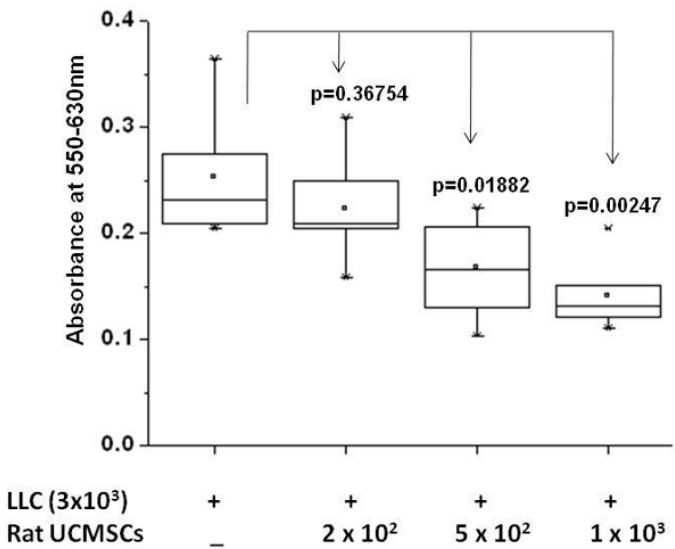

Figure 1 Direct co-culture of a small number of rat UCMSCs significantly attenuated the growth of LLC cells. Rat UCMSCS (300, 500 and 1000 cells/well) were co-cultured with $3 \times 10^{3}$ LLC (rat UCMSCs: LLC ratio, 1:10, 1:6 and 1:3). After three days of coculture, the MTT assay was carried out. Panel A shows the morphology of LLC alone or LLC co-cultured with rat UCMSCs for 72 hrs. Scale bars in the pictures indicate $1000 \mu \mathrm{m}$. Panel B shows the summarized results from the MTT assay. The experiment was performed twice with 6 determinations at each point.

corroborate with the results from the MTT and thymidine uptake assays. Un-engineered rat UCMSCs dosedependently attenuated the total number of LLC cells. This Transwell experiment suggests that rat UCMSCdependent cell growth attenuation may be mediated through a diffusible molecule or molecules produced by rat UCMSCs. Another Transwell cell culture in which LLC cells $\left(1 \times 10^{5}\right.$ cells $)$ were cultured in the inserts and rat UCMSCs $\left(1.7 \times 10^{3}\right)$ were cultured in the bottom of the wells revealed that LLC cells do not alter the growth of rat UCMSCs (data not shown).

Since anchorage-independent growth is a hallmark of tumorigenesis, a soft agar assay was carried out to evaluate the effect of rat UCMSCs on the growth of LLC cell 


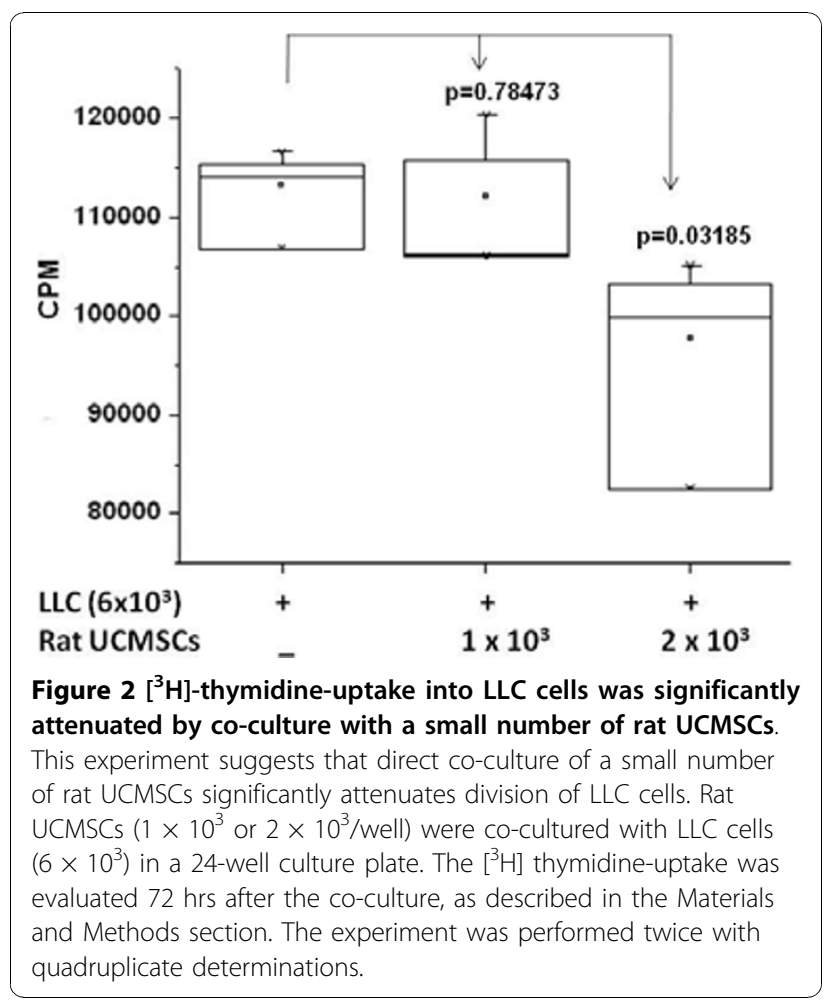

\section{A}
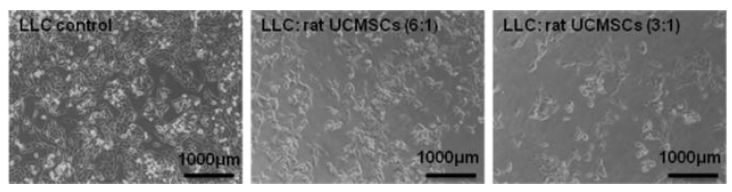

B

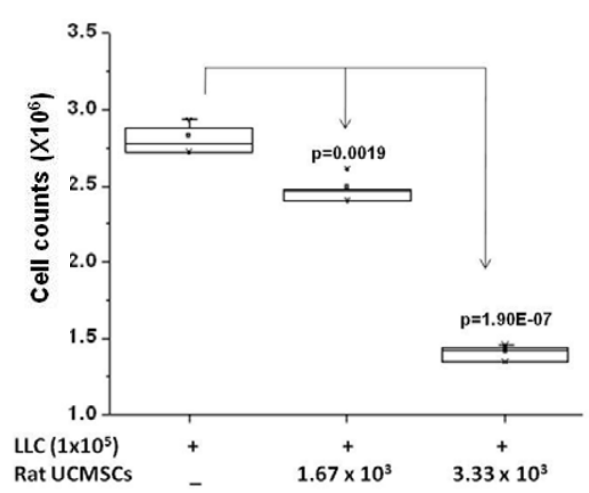

Figure 3 Rat UCMSCs significantly attenuated the growth of LLC cells in a Transwell co-culture. This experiment suggests that indirect co-culture of a small number of rat UCMSCs significantly attenuates the growth of LLC cells. Panel A shows the morphology of LLC alone or LLC indirectly co-cultured with rat UCMSCs for 72 hrs. Panel B shows the summarized results from direct cell counts using a hemocytometer. The experiment was performed twice with triplicate determinations. colonies. The soft agar colony assay is an in vitro model to mimic in vivo xenograft models. The results showed that LLC cell colony size and number in soft agar were significantly attenuated when rat UCMSCs were cultured in the bottom of the culture dish (Figure 4). Since LLC cells were separated from rat UCMSCs by a solid agar layer (approximately $1 \mathrm{~mm}$ thickness), and the two cell types physically do not contact each other, the colony assay results also suggest that rat UCMSCdependent growth attenuation was mediated through molecules produced by rat UCMSCs which diffused to LLC cells.

\section{Rat UCMSCs induced G0/G1 cell cycle arrest}

The effect of rat UCMSCs on the cell cycle of LLC cells was evaluated by flow cytometry after the LLC cells were co-cultured with rat UCMSCs in Transwell culture dishes. The result shows that rat UCMSCs caused G0/ G1 arrest of LLC cells, thus increasing the G0/G1 population and decreasing the $S$ phase population of LLC cells (Figure 5A). As shown in Figure 5B, the G0/G1 phase cell populations (\%) in LLC alone, LLC:UCMSCs (6:1), and LLC:UCMSCs (3:1) were $39.02 \pm 0.06,39.89 \pm$ 0.11 , and $45.84 \pm 0.81$, respectively; S phase populations (\%, same order as above) were $49.35 \pm 0.24,49.69 \pm$ 0.25 , and $41.33 \pm 0.63$, respectively. Furthermore, cyclins

A
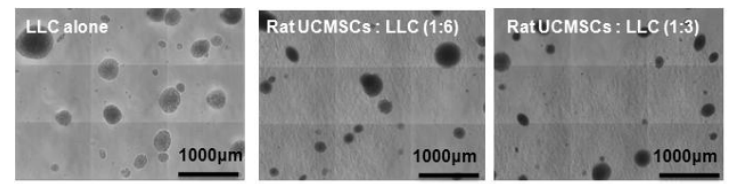

B

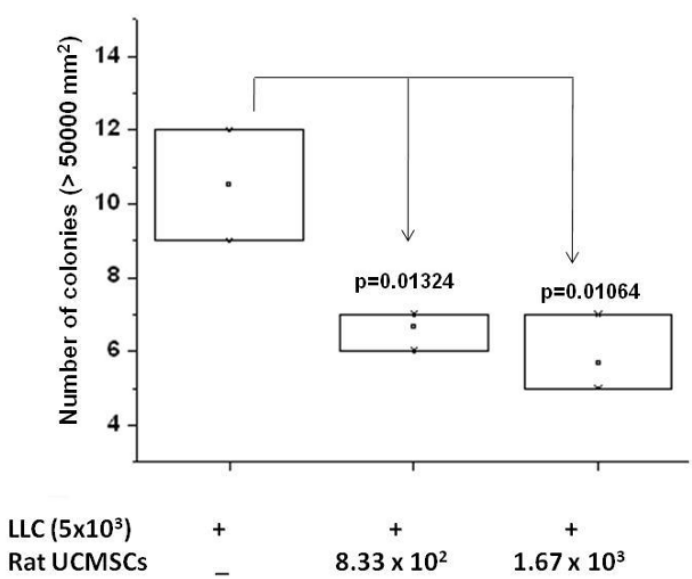

Figure 4 Rat UCMSCs significantly attenuated colony growth of LLC cells. Panel A shows the morphology of colonies of LLC alone or LLC co-cultured with rat UCMSCs in soft agar after 16 days. Panel $B$ shows the summarized results from the colony assay. The experiment was performed twice with triplicate determinations. 


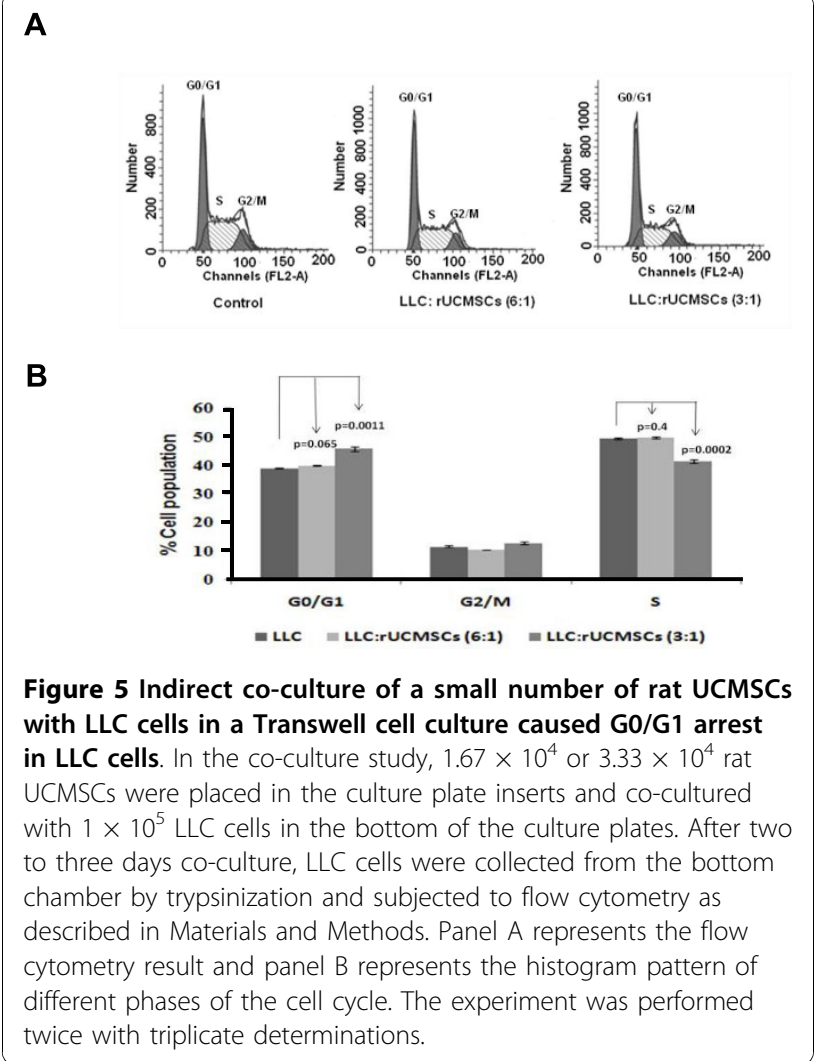

and cyclin-dependent kinase were analyzed by Western blot analysis. As shown in Figure 6, co-culture with rat UCMSCs significantly attenuated protein expression levels of cyclin A and CDK2, but not cyclin E. These results also indicate cell cycle arrest in G0/G1 phase.

\section{Un-engineered rat UCMSCs significantly attenuate growth of LLC grafts in syngeneic mice}

We tested the growth attenuation ability of unengineered naïve rat UCMSCs on the lung LLC tumors by local (intratracheal) and systemic (IV) administration. Results indicate that a single intratracheal administration of rat UCMSCs 5 days after LLC transplantation almost completely inhibited tumor growth as measured by tumor weight (Figure 7A). The IV administration of rat UCMSCs also significantly attenuated tumor growth despite the fact that tumor growth was more prominent due to a larger number of the LLC cells transplanted (2 million cells/mouse) (Figure 7B). Histological analysis revealed that lungs of PBS injected mice were occupied by large amounts of cancer tissue, whereas rat UCMSCtreated groups contained almost negligible (intratracheal injection group) or very small amounts of tumor mass (systemic injection group). Fluorescence microscopic observation of serial sections used for H\&E staining shows the engraftment of rat UCMSCs in close

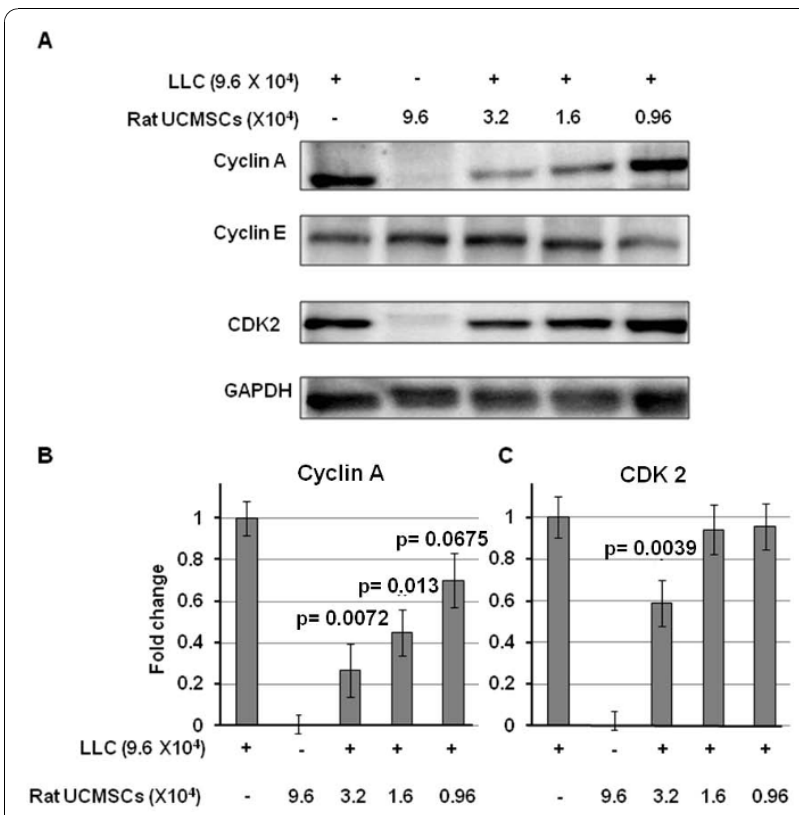

Figure 6 Rat UCMSCs significantly attenuated protein expression of both cyclin A (Panels A and B) and CDK2 (Panels $A$ and $C$ ), but not cyclin E (Panel $A$ ), in co-cultured LLC cells.

This experiment suggests that indirect co-culture of a small number of rat UCMSCs caused G0/G1 arrest in LLC cells. Samples were prepared as described in the Figure 5 legend and in Materials and Methods, and subjected to Western blot analysis. Sample preparation and Western blot analysis were performed three times with duplicate determinations. The pictures (Panel A) represent typical blotting results. $P$ values were calculated as compared to the level of control.

proximity to or within tumor tissues of tumor bearing mice that received SP-DiI labeled rat UCMSCs either intratracheally or systemically (Figure 8). However, SPDiI labeled rat UCMSCs were not detected in normal areas of the lung.

\section{Rat UCMSC treatment markedly increased apoptosis in tumor tissues}

To evaluate the effect of the rat UCMSCs on the apoptotic activities of tumor cells in vivo, the percentage of TUNEL-positive cell area in the tumors was determined. The percentage of TUNEL-positive cell area was two times higher in rat UCMSC treated tumors than in PBS treated control tumors (Figure 9). These results indicated that treatment with un-engineered rat UCMSCs increases apoptosis significantly.

\section{Discussion}

The present study shows for the first time that naïve rat UCMSCs have the potential to attenuate rapidly growing murine lung carcinoma cells in a syngeneic mouse model. This effect was observed in most tumor-bearing mice transplanted either locally or systemically with rat 


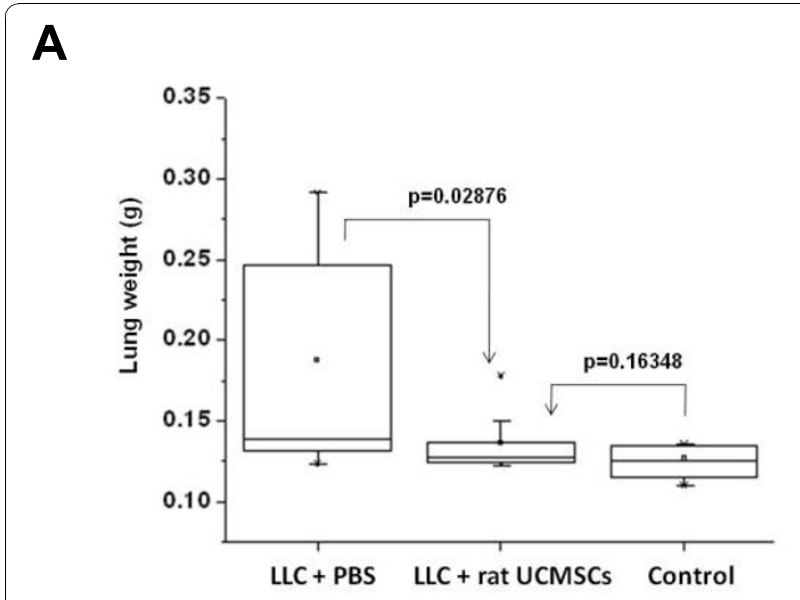

B

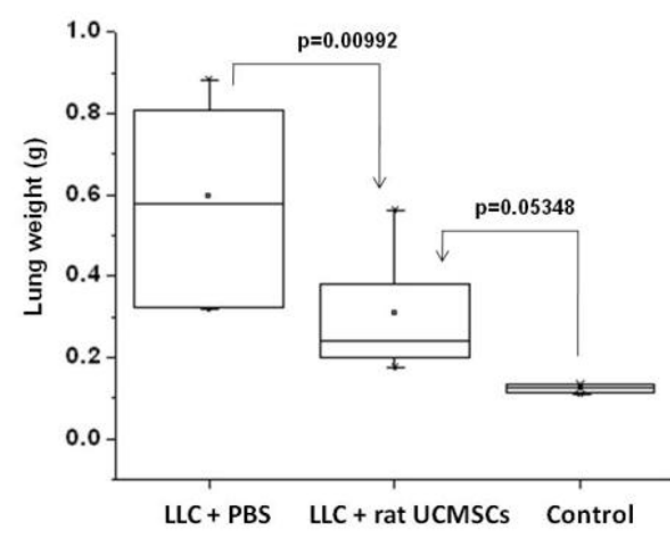

Figure 7 Administration of rat UCMSCs significantly attenuated the growth of LLC tumors in a syngeneic mouse model. Panel A shows that lung weight in mice transplanted with one million LLC cells declines almost to non-cancerous control levels after a single intratracheal administration of rat UCMSCs ( 0.35 million cells). Panel B shows that systemic transplantation of rat UCMSCs (one million cells twice) also significantly decreased the weight of the LLC-bearing lungs despite a marked increase of tumor burden due to a larger number of LLC cells transplanted (two million cells). In both experiments, eight mice per group were used except for the rat UCMSCs intratracheally administered group, which consisted of nine mice.

UCMSCs. In addition, we describe here the following important new findings: 1 . in co-culture, a relatively small number of rat UCMSCs significantly attenuated proliferation of LLC cells; 2 . in vitro three dimensional LLC colony formation is markedly attenuated when rat UCMSCs are present as a monolayer in the bottom of the culture; 3) rat UCMSCs caused G0/G1 arrest in LLCs, and this may be a possible mechanism of growth attenuation; 4) either local or systemic rat UCMSC therapy significantly decreased the lung tumor burden; 5) rat UCMSCs administered intratracheally or IV 'home' into or near the lung tumors

Attenuation of tumor cell growth by rat UCMSCs appears to have both contact-independent and -dependent components. Interestingly, Khakoo et al. showed that bone marrow mesenchymal stem cells could only mediate their effect on Kaposi sarcoma by contact with the tumor cells in vitro [17]. Here we showed that in co-culture assays (Figures. 1 and 2); rat UCMSCs cocultured with LLC cells significantly attenuate LLC cell proliferation. Since the number of rat UCMSCs was significantly smaller than LLCs (1:6 or $1: 3)$, this rat UCMSC-dependent effect is not strictly contactmediated; it is likely that some factor(s) independent of cell-to-cell contact is involved. In the Transwell coculture study and soft agar colony assay, in which LLC cells were not in direct contact with rat UCMSCs (Figures. 3 and 4), the LLC growth inhibition by rat UCMSCs implies involvement of a diffusible factor or factors secreted by the stem cells. This implication is in good agreement with previous studies from our laboratory in which rat UCMSCs attenuated growth of rat mammary carcinoma cells, apparently through diffusible molecules [15]. This factor may be associated with the regulation of the cell cycle, since the present study clearly indicated that rat UCMSCs induced G0/G1 arrest in LLC cells when they were co-cultured in a Transwell culture system (Figure 5), in which the two cell types do not have direct contact. Furthermore, the anti-tumor effect shown here may also be associated with pro-apoptotic factors produced by naïve rat UCMSCs (Figures. 6 and 9). Based on the present study, cell cycle regulationand/or apoptosis-associated genes may be good candidates. However, an involvement of other mechanisms, such as a stimulation of the host immune system, may also be a part of tumor growth attenuation in vivo.

The homing ability of stem cells has previously been exploited for drug delivery and targeted gene delivery $[5,6,8-10,12,18]$. The homing of stem cells to tumors and other areas of inflammation is well established $[5-8,19]$. The homing ability of stem cells appears to be mediated by chemokines secreted by the tumors or their associated stroma [20-22]. Potential chemokines could include growth factors such as platelet derived growth factor (PDGF) family members and epidermal growth factor (EGF) [23] in addition to classical chemoattractants such as CXCR4 and its ligand, SDF-1. In the present study, SP-DiI-loaded rat UCMSCs were detected either within the tumors or adjacent to the tumors (Figure 8). Potential reasons why only a few dye loaded UCSMCs were detected in lung tumor sites follow: 1) lung tissue was fixed with $10 \%$ formalin, and formalin is known to have a fluorescence quenching effect; 2 ) only $20 \%$ of UCMSCs were labeled by SD-DiI; and 3) the 

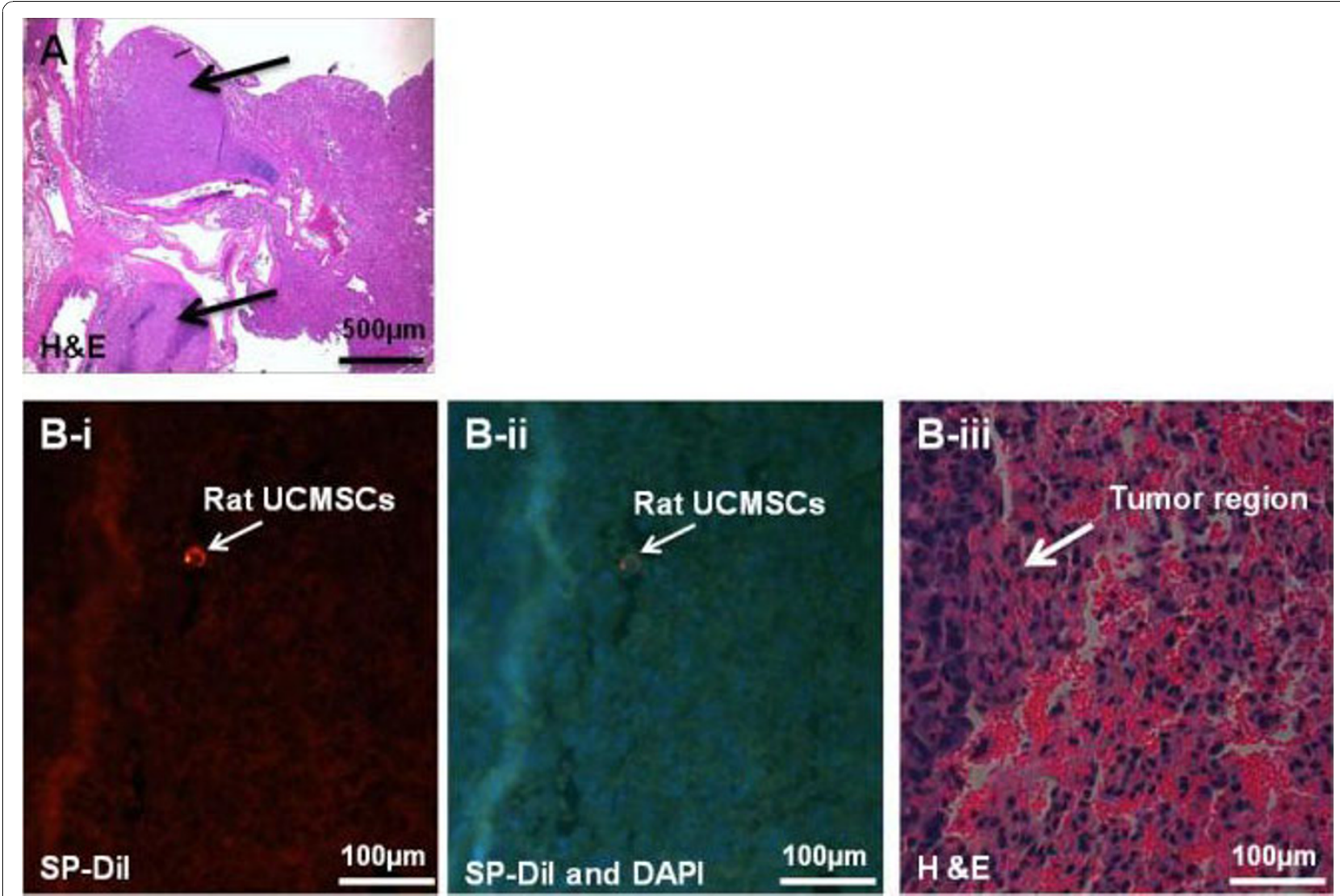

Figure 8 SP-Dil labeled rat UCMSCs administered intratracheally were detected near the tumors. This experiment suggests that intratracheally administered rat UCMSCs home at adjacent to the lung tumor area. Serial sections from paraffin-embedded lung were used for the detection of rat UCMSCs labeled with SP-Dil fluorescent dye either with SP-Dil fluorescence alone (B-i) or with both DAPI nuclear staining and SP-Dil fluorescence (B-ii). Tumor morphology was observed after H\&E staining (A, PBS-treated tumors, arrows indicate tumors, and B-iii, rat UCMSC-treated tumor, arrow indicates tumor margin). Scale bars in Panel A and B indicate $500 \mu \mathrm{m}$ and $100 \mu \mathrm{m}$, respectively.

chances of encountering a small number of UCMSCs in a $4 \mu \mathrm{m}$ section are relatively low even if a large number of cells were localized in the entire tumor area. Thus, detection of even a small number of SP-DiI-loaded rat UCMSCs in or adjacent to the tumors may imply that rat UCMSCs were attracted to the chemokines produced by tumor tissues and homed to the tumor tissues.

The ability of naïve UCMSCs to eliminate lung adenocarcinomas is a distinct advantage, since any manipulation causing the cells to express an exogenous gene could alter them in some way that would potentially make them less safe as transplantable cells. However, since rat UCMSCs are not directly applicable to human therapy, key mechanism(s) by which rat UCMSCs exhibit their powerful anti-tumor effect should be identified first. This mechanism may be applied to human UCMSCs for future human application. Therefore, it will be worthwhile to identify which genes or gene products make rat UCMSCs so powerful for attenuation of lung cancer growth. If human UCMSCs are as potent as rat UCMSCs, human UCMSCs will potentially be utilized for human cancer therapy. If human UCMSCs are not as potent as rat UCMSCs, their cytotoxicity to cancer cells may be enhanced by manipulation of their gene expression based on the study of rat UCMSCdependent cytotoxicity against lung cancer.

In this study we have used rat origin UCMSCs for a mouse cancer model and they have shown a very strong effect. Whether the rat UCMSC-dependent anti-tumorigenetic effect is partially due to the xenotransplantation is unclear. However, since rat UCMSCs were extremely potent in cell growth attenuation in simple in vitro cell culture studies where no immune cells or immunoglobulin were involved, it is likely that their in vivo effect is also independent of nonspecific immune surveillance induced by xenotransplantation. In support of this, histopathological analysis did not show lymphocyte infiltration in tumor tissues (data not shown). In addition, our previous finding that human UCMSCs are poorly immunogenic [14] also indirectly supports the above speculation.

Naïve UCMSCs have many potential advantages for cytotherapy. Among many tissue-originated multipotent 

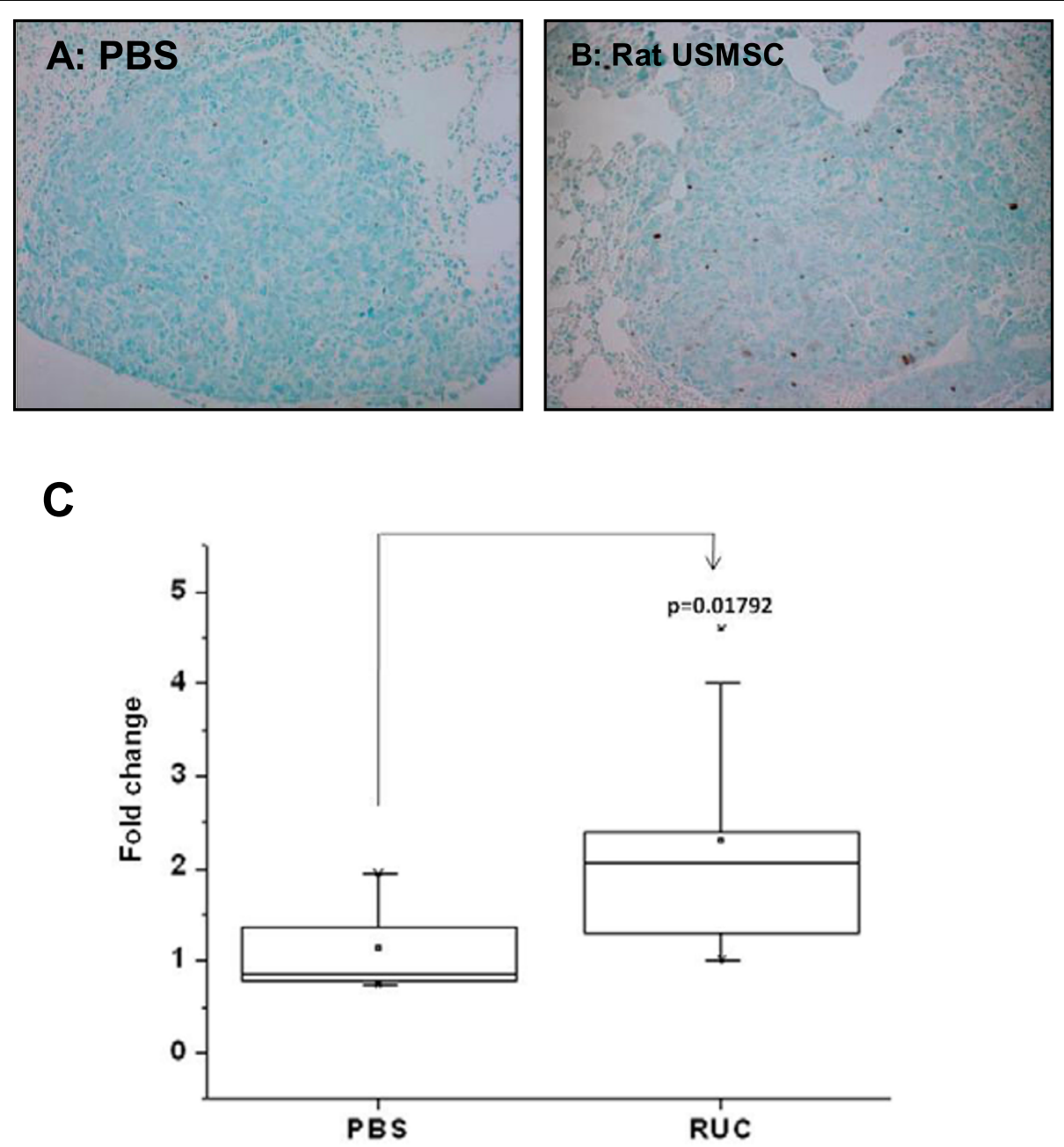

Figure 9 Analysis of apoptosis in LLC tumors in syngeneic mouse lungs treated with either PBS (A) or rat UCMSCs (B) indicates that rat UCMSCs increase apoptotic cell death. Lungs were collected, and apoptosis in tumors was analyzed by evaluating the area of TUNEL positive cells (panels A and B) as described in Materials and Methods. The average TUNEL positive area(C) were determined by analyzing ten tumor areas in each treatment group and expressed as fold change compared to PBS-treated controls.

stem cells, UCMSCs are very usable due to their abundance, low immunogenicity [14], lack of CD34 and CD45 expression, and simplicity of the methods for harvest and in vitro expansion [12,24,25]. These properties of UCMSCs encourage their development as therapeutic tools or agents because they can potentially be used for allogeneic transplantation.

\section{Conclusions}

In in vitro studies, co-culture of rat UCMSCs significantly attenuated the proliferation of LLC cells as monitored by MTT assay, thymidine uptake, and direct cell counts. Rat UCMSCs also markedly reduced LLC colony size and number. Co-culture of rat UCMSCs caused G0/ G1 arrest of LLC cells. This is evident in the decrease of cyclin A and CDK2 expression. The in vivo studies showed that rat UCMSC treatment significantly decreased tumor weight and the total tumor area. Histological study revealed that intratracheally or systemically administered rat UCMSCs homed to tumor areas and survived for at least 3 weeks without any evidence of differentiation or adverse effects. Thus the findings described here suggest that UCMSCs may represent a new therapeutic modality for the treatment of lung cancer and will have important implications for patients with lung cancer and other types of cancer.

\section{List of abbreviations}

DAPI: 4',6-diamidino-2-phenylindole; EGF: epidermal growth factor; FBS: fetal bovine serum; H\&E: hematoxyline and eosin; IFN- $\beta$ : Interferon beta; ITS-X: insulin-transferrin-selenium-X; LLC: Lewis lung carcinoma; MTT: Methylthiazol Tetrazolium; PDGF: platelet derived growth factor; PDGF-BB: platelet derived growth factor-BB; SDF-1: stroma derived factor-1; UCMSCs: umbilical cord 
matrix stem cells; CDK2: Cyclin-dependent Kinase 2; GAPDH: Glyceraldehyde 3-phosphate dehydrogenase;

\section{Acknowledgements}

This work was supported by Kansas State University (KSU) Terry C. Johnson Center for Basic Cancer Research, Kansas Bioscience Authority Collaborative Cancer Research Initiative grant, KSU Targeted Excellence research grant, Kansas State Legislative Appropriation, KSU College of Veterinary Medicine Dean's fund, NIH grants P20 RR017686, P20 RR01556 and R21 CA135599 and Joan's Legacy Foundation.

\section{Authors' contributions}

$\mathrm{DKM}, \mathrm{CD}, \mathrm{AK}, \mathrm{CK}, \mathrm{ZW}$, and $\mathrm{MT}$ were responsible for the study design, experimental work, data evaluation and analysis, and drafting the manuscript. MMP and DT were consulted extensively in the experimental design and interpretation of results, as well as in the preparation of the manuscript. MT was the research supervisor and participated in the study design, assessment of the results, and drafting the manuscript. All authors read and approved the manuscript.

\section{Competing interests}

The authors declare that they have no competing interests.

Received: 25 September 2009 Accepted: 28 October 2010

Published: 28 October 2010

\section{References}

1. Jemal A, Siegel R, Ward E, Hao Y, Xu J, Thun MJ: Cancer Statistics, 2009. CA Cancer J Clin 2009

2. Johnson $\mathrm{DH}$, Schiller JH: Novel therapies for the treatment of non-small cell lung cancer. Cancer Chemother Biol Response Modif 2002, 20:763-786.

3. Lynch TJ, Adjei AA, Bunn PA Jr, DuBois RN, Gandara DR, Giaccone G, Govindan R, Herbst RS, Johnson BE, Khuri FR, et al: Novel agents in the treatment of lung cancer: conference summary statement. Clin Cancer Res 2004, 10(12 Pt 2):4199s-4204s.

4. Corsten MF, Shah K: Therapeutic stem-cells for cancer treatment: hopes and hurdles in tactical warfare. Lancet Oncol 2008, 9(4):376-384.

5. Aboody KS, Brown A, Rainov NG, Bower KA, Liu S, Yang W, Small JE, Herrlinger U, Ourednik V, Black PM, et al: Neural stem cells display extensive tropism for pathology in adult brain: evidence from intracranial gliomas. Proc Natl Acad Sci USA 2000, 97(23):12846-12851.

6. Nakamizo A, Marini F, Amano T, Khan A, Studeny M, Gumin J, Chen J, Hentschel S, Vecil G, Dembinski J, et al: Human bone marrow-derived mesenchymal stem cells in the treatment of gliomas. Cancer Res 2005, 65(8):3307-3318.

7. Rachakatla RS, Marini F, Weiss ML, Tamura M, Troyer D: Development of human umbilical cord matrix stem cell-based gene therapy for experimental lung tumors. Cancer Gene Ther 2007, 14(10):828-835.

8. Studeny M, Marini FC, Champlin RE, Zompetta C, Fidler IJ, Andreeff M: Bone marrow-derived mesenchymal stem cells as vehicles for interferon-beta delivery into tumors. Cancer Res 2002, 62(13):3603-3608.

9. Studeny M, Marini FC, Dembinski JL, Zompetta C, Cabreira-Hansen M, Bekele BN, Champlin RE, Andreeff M: Mesenchymal stem cells: potential precursors for tumor stroma and targeted-delivery vehicles for anticancer agents. J Natl Cancer Inst 2004, 96(21):1593-1603.

10. Aboody KS, Najbauer J, Schmidt NO, Yang W, Wu JK, Zhuge Y, Przylecki W, Carroll R, Black PM, Perides G: Targeting of melanoma brain metastases using engineered neural stem/progenitor cells. Neuro Oncol 2006, 8(2):119-126.

11. Kumar S, Chanda D, Ponnazhagan S: Therapeutic potential of genetically modified mesenchymal stem cells. Gene Ther 2008, 15(10):711-715.

12. Mitchell KE, Weiss ML, Mitchell BM, Martin P, Davis D, Morales L, Helwig B, Beerenstrauch M, Abou-Easa K, Hildreth T, et al: Matrix cells from Wharton's jelly form neurons and glia. Stem Cells 2003, 21(1):50-60.

13. Cho PS, Messina DJ, Hirsh EL, Chi N, Goldman SN, Lo DP, Harris IR, Popma SH, Sachs DH, Huang CA: Immunogenicity of umbilical cord tissue derived cells. Blood 2008, 111(1):430-438.

14. Weiss ML, Anderson C, Medicetty S, Seshareddy KB, Weiss RJ, VanderWerff I, Troyer D, McIntosh KR: Immune properties of human umbilical cord Wharton's jelly-derived cells. Stem Cells 2008, 26(11):2865-2874.
15. Ganta C, Chiyo D, Ayuzawa R, Rachakatla R, Pyle M, Andrews G, Weiss M, Tamura M, Troyer D: Rat umbilical cord stem cells completely abolish rat mammary carcinomas with no evidence of metastasis or recurrence 100 days post-tumor cell inoculation. Cancer Res 2009, 69(5):1815-1820.

16. Ayuzawa A, Doi C, Rachakatla RS, Pyle MM, Maurya DK, Troyer D, Tamura M: Naive human umbilical cord matrix derived stem cells significantly attenuate growth of human breast cancer cells in vitro and in vivo. Cancer letters 2009, 280(1):31-37.

17. Khakoo AY, Pati S, Anderson SA, Reid W, Elshal MF, Rovira II, Nguyen AT, Malide D, Combs CA, Hall G, et al: Human mesenchymal stem cells exert potent antitumorigenic effects in a model of Kaposi's sarcoma. J Exp Med 2006, 203(5):1235-1247.

18. Aboody KS, Najbauer J, Danks MK: Stem and progenitor cell-mediated tumor selective gene therapy. Gene Ther 2008, 15(10):739-752.

19. Hall $B$, Andreeff M, Marini F: The participation of mesenchymal stem cells in tumor stroma formation and their application as targeted-gene delivery vehicles. Handb Exp Pharmacol 2007, , 180: 263-283.

20. Muller A, Homey B, Soto H, Ge N, Catron D, Buchanan ME, McClanahan T, Murphy E, Yuan W, Wagner SN, et al: Involvement of chemokine receptors in breast cancer metastasis. Nature 2001, 410(6824):50-56.

21. Lee BC, Lee TH, Avraham S, Avraham HK: Involvement of the chemokine receptor CXCR4 and its ligand stromal cell-derived factor 1alpha in breast cancer cell migration through human brain microvascular endothelial cells. Mol Cancer Res 2004, 2(6):327-338.

22. Karnoub AE, Dash AB, Vo AP, Sullivan A, Brooks MW, Bell GW, Richardson AL, Polyak K, Tubo R, Weinberg RA: Mesenchymal stem cells within tumour stroma promote breast cancer metastasis. Nature 2007, 449(7162):557-563.

23. Arbab AS, Janic B, Knight RA, Anderson SA, Pawelczyk E, Rad AM, Read EJ, Pandit SD, Frank JA: Detection of migration of locally implanted AC133+ stem cells by cellular magnetic resonance imaging with histological findings. Faseb J 2008, 22(9):3234-3246.

24. Weiss ML, Medicetty S, Bledsoe AR, Rachakatla RS, Choi M, Merchav S, Luo Y, Rao MS, Velagaleti G, Troyer D: Human umbilical cord matrix stem cells: preliminary characterization and effect of transplantation in a rodent model of Parkinson's disease. Stem Cells 2006, 24(3):781-792.

25. Weiss ML, Troyer DL: Stem cells in the umbilical cord. Stem Cell Rev 2006, 2(2):155-162.

\section{Pre-publication history}

The pre-publication history for this paper can be accessed here: http://www.biomedcentral.com/1471-2407/10/590/prepub

doi:10.1186/1471-2407-10-590

Cite this article as: Maurya et al:: Therapy with un-engineered naïve rat umbilical cord matrix stem cells markedly inhibits growth of murine lung adenocarcinoma. BMC Cancer 2010 10:590.

\section{Submit your next manuscript to BioMed Central and take full advantage of:}

- Convenient online submission

- Thorough peer review

- No space constraints or color figure charges

- Immediate publication on acceptance

- Inclusion in PubMed, CAS, Scopus and Google Scholar

- Research which is freely available for redistribution

Submit your manuscript at www.biomedcentral.com/submit
C Biomed Central 